maintenance therapy, secondary loss of response is common affecting approximately $10 \%-15 \%$ of patients/year. Increasing drug levels, whether by doubling the dose or decreasing the interval, recaptures response in approximately $75 \%$ of patients. However, both of these strategies have cost implications. Anecdotal reports suggest that in patients losing response on maintenance infliximab (IFX) $5 \mathrm{mg} / \mathrm{kg}$, a temporary increase to double doses (DD; $10 \mathrm{mg} / \mathrm{kg}$ ) can lead to subsequent recapture of response at the lower dose. In a small cohort, we have previously shown that this strategy was not viable in the majority of patients. We now present an extended cohort across two centres with longer follow-up.

Methods We performed a retrospective review across two tertiary centres of all patients with Crohn's disease who had received temporary increases to DD of IFX for loss of response. Demographic data, HBI prior to the first infusion at the higher dose and prior to the first infusion at the lower dose, and ability to continue on IFX at $5 \mathrm{mg} / \mathrm{kg}$ were recorded.

Results 34 patients (18M:16F, median age 24 (range 12-51)) received DD IFX for loss of response. Median disease duration was 3 years (range $0-32$ ) and the median time to dose increase from starting IFX was 12 months (range 3-60). All had received standard induction doses of IFX at $5 \mathrm{mg} / \mathrm{kg}$ on weeks 0,2 and 6 and were on scheduled maintenance therapy. The dose interval prior to dose increase was 8 weeks for 24 patients, 7 weeks for 1 , and 6 weeks for 9. 26 patients were on concurrent immunomodulators and 8 were not. One patient received $4 \mathrm{DD}, 27$ received $2 \mathrm{DD}$ and six patients 1 DD. Dose increase was effective in the short term with the median HBI falling from 6 (range $0-27$ ) prior to the first infusion at $10 \mathrm{mg} /$ $\mathrm{kg}$, to $1(0-7)$ prior to the first infusion back at the standard dose $(5 \mathrm{mg} / \mathrm{kg})(\mathrm{p}=0.003)$. However, only seven patients remained on IFX at the end of follow-up (median 13 months (range 8-25)). The median interval to discontinuing IFX was 4 months after the first DD (1-19). Of those who discontinued, four had infusion reactions while the others failed to maintain a response to IFX.

Conclusion Because of the limited treatment options available in Crohn's disease, attempting to recapture response in patients on IFX is appropriate. However, while temporary double-dosing is effective in the short term, it does not deliver long term disease control after subsequent dose reduction. Whether dose reduction is possible after prolonged dose increase remains to be answered.

Competing interests None declared.

\section{PTU-129 INITIAL EXPERIENCE WITH INFLIXIMAB LEVELS IN A TERTIARY IBD CENTRE}

doi:10.1136/gutjnl-2012-302514c.129

${ }^{1} \mathrm{P} M$ Irving, * ${ }^{2} \mathrm{Z}$ Arkir, ${ }^{1} \mathrm{~J}$ Duncan, ${ }^{1} \mathrm{M}$ Sastrillo, ${ }^{1} \mathrm{~S}$ Anderson, ${ }^{1} \mathrm{~J}$ Sanderson.

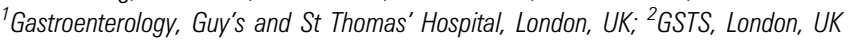

Introduction Biologic use in the UK is increasing in patients with Crohn's disease (CD). While highly effective at inducing and maintaining remission, secondary loss of response occurs in approximately $15 \%$ of patients on maintenance treatment with biologics every year. There is some preliminary evidence that drug levels may play a role in this. However, measurement of anti-TNF levels has not been routinely available in the UK until very recently. Methods We performed a service evaluation of a CE marked kit measuring serum levels of infliximab (IFX) as well as anti-drug antibodies (BMD, Marne La Vallee, France). Samples were taken immediately prior to infusion of IFX in patients attending for infusions over a 9-week period (March-May 2011). Results were not used in clinical management. A retrospective notes review was undertaken in January 2012 to see how drug levels related to clinical activity and to outcome. Only patients with CD on stable maintenance therapy were included and if repeated samples were taken, only the first measurement was used.
Results 52 samples were taken on 41 patients. Eight were excluded either because they did not have CD or because they were still in the induction phase of treatment. 33 patients ( 17 male) were, therefore, included in the analysis. Seven had subtherapeutic levels (SL) of IFX $(<2 \mu \mathrm{g} / \mathrm{ml})$. Patients with SL had a higher median Harvey Bradshaw index (HBI) (5 (range $0-7)$ ) and $\mathrm{C}$ reactive protein (CRP) $(8(0-50))$ at the time the sample was taken than those with therapeutic levels (TL) (HBI $(1(0-8) p=0.03$ : CRP $0(0-8) p<0.05)$. The highest HBI and CRP recorded during subsequent follow-up was also higher in those with SL than TL but not significantly so (HBI SL 4 (2-11) vs TL $2(0-12)$ : CRP SL $11(0-56)$ vs TL $0(0-21))$. Patients with SL were also more likely to require dose escalation of IFX (SL 2/7: TL 0/ $26(p<0.04))$ and intervention (change of drug therapy or surgical/ endoscopic intervention) (SL 3/7: TL 2/27 ( $p=0.05)$ ).

Conclusion Subtherapeutic IFX levels were associated with increased disease activity defined by biomarkers (CRP) and disease activity scores (HBI). Subtherapeutic levels were also predictive of a worse disease course over the following 6-8 months. Measuring anti-TNF drug levels in patients with IBD is promising and the utility of these tests in every day practice should be investigated further.

Competing interests None declared.

\section{PTU-130 MUCOSAL MRNA EXPRESSION PROFILING FROM THE TERMINAL ILEUM AND COLON REVEALS UNDER EXPRESSION OF CLAUDIN 8, A TIGHT JUNCTION MOLECULE, AS POTENTIALLY CAUSAL IN ULCERATIVE COLITIS}

doi:10.1136/gutjnl-2012-302514c.130

${ }^{1} \mathrm{P}$ J Smith, ${ }^{*}{ }^{1} \mathrm{~A}$ P Levine, ${ }^{1} \mathrm{G} W$ Sewell, ${ }^{1} \mathrm{~N}$ R O'Shea, ${ }^{2} \mathrm{R}$ Vega, ${ }^{2} \mathrm{~S}$ L Bloom, ${ }^{1} \mathrm{~A}$ M Smith, ${ }^{1} \mathrm{~A}$ W Segal. ${ }^{1}$ Department of Medicine, University College London, London, UK; ${ }^{2}$ Department of Gastroenterology, University College London Hospital, London, UK

Introduction Intestinal barrier dysfunction plays an important role in the pathogenesis of ulcerative colitis (UC). We investigated mRNA profiles of mucosa from the colon and terminal ileum, in patients with UC and controls (HC) to identify genes that might be implicated in the pathogenesis of the disease.

Methods Mucosal biopsies were taken from 24 quiescent UC patients (Mayo score $<3$ ) and $33 \mathrm{HCs}$ undergoing colonoscopy. Patients were on no treatment or on 5-aminosalicylates \pm azathioprine. HCs were patients without organic disease. Parallel biopsies were taken for RNA extraction and histology from macroscopically non-inflamed mucosa in the terminal ileum (TI), ascending, descending and sigmoid colon, and rectum. cRNA was hybridised to Illumina HumanHT v12.0 Expression Beadchips. Expression data were log transformed and normalised. Probes with a detection $p$ value $<0.01$ were analysed. Comparing 85 biopsies from HCs and 68 biopsies from UC patients across the colon, the data for each bowel location were adjusted to the mean $\mathrm{HC}$ rectal expression level. Where multiple biopsies were taken from the same individual, the adjusted data across all biopsies for that individual were averaged. $T$ tests between groups and outlier analysis $(p<0.005$, fold change $(\mathrm{FC}) \geq 1.5$ ) were performed using proprietary software.

Results Of the $\sim 30 \mathrm{~K}$ probes analysed, the two most significantly under-expressed in UC in the colon were those of claudin 8 (CLDN8) with FCs $2.94\left(p=1.29 \times 10^{-5}\right)$ and $3.45\left(p=3.92 \times 10^{-5}\right)$. The expression of claudin 8 increased distally in the colon, whereas claudins 3, 7 and 23 were highly, and uniformly, expressed throughout the colon and were normal in UC. Outlier analysis between HC and UC showed CLDN8 to be significantly underexpressed in $25 \%>40 \%$ of UC patients at all 4 colonic sites. There were no CLDN8 UC outliers in the TI. Most of these outlier patients demonstrated consistent levels of under-expression 
throughout the colon, and their histology revealed microscopic inflammation. Other patients with histologically active disease had normal CLDN8 expression.

Conclusion CLDN8 is significantly under-expressed in the UC colon. Outlier analysis has also identified a group of patients in whom CLDN8 is grossly under-expressed. Low expression of CLDN8 in UC could be secondary to inflammation, although the evidence presented here is against this. Reduced levels of CLDN8 could lead to a weak and permeable mucosa predisposing to UC by reducing barrier resistance and allowing penetration by microbes.

Competing interests None declared.

\section{Adolescent and young people

PTU-131 RESPONSE TO ENTERAL NUTRITION PREDICTS
INCREASED LENGTH OF REMISSION IN CHILDREN WITH
CROHN'S DISEASE

doi:10.1136/gutjnl-2012-302514c.131

A Rao, ${ }^{*}$ N Kamperidis, Y Koodun, S Naik, N M Croft, I R Sanderson. Centre for Digestive Diseases, Barts and the London School of Medicine and Dentistry, Queen Mary University of London, London, UK

Introduction Exclusive enteral nutrition (EEN) is the primary therapy for children with intestinal Crohn's disease (CD) in the UK. We hypothesised that entering remission with EEN predicted a longer duration of remission.

Methods Retrospective data were obtained on children with CD from 2003 to 2006 at a tertiary paediatric gastroenterology centre. Response was determined by Physicians Global Assessment. Outcome measures investigated were: relapse rates, time to relapse, corticosteroid (CS) use and treatment escalation. Relapse was defined as worsening symptoms and/or increase in CRP with a change in medication. $p$ Values of $<0.05$ were considered significant.

Results 75 children with CD were diagnosed between 2003 and 2006, in whom 62 had 5 year follow-up data available. 56 patients $(90.3 \%)$ received EEN upon diagnosis. The others received 5-ASA [4] or antibiotics [2], and were excluded from the analysis. No patients received corticosteroids as initial treatment. The median age [range] at diagnosis was 12.87 [4.84-15.86] years. 62.5\% [35] of patients had ileo-colonic disease. $94.6 \%$ [53/56] of patients tolerated EEN. $57.1 \%$ [32] of patients went into clinical remission with EEN. Corticosteroids were prescribed to those who failed to enter remission with EEN. Multivariate analysis showed no correlation between disease location $(p=0.70)$, ethnicity $(p=0.43)$, age $(p=0.25)$ or CRP $(p=0.73)$ and response to EEN. All of the patients with colonic disease relapsed over 5 years $(n=7)$, compared to $79 \%[11 /$ 14] of patients with ileal disease and 77\% [27/35] of patients with ileo-colonic disease $(p=0.37)$. The patients who responded to EEN remained in remission significantly longer than the non-responders. Median time to relapse [range] over the 5 years was 17.4 [4.23-49.32] months in responders vs 9.72 [2.87-47.6] months in non-responders; $p=0.041$ (Abstract PTU-131 figure 1). 50\% [16/32] of patients who responded to EEN had no corticosteroid use over the 5 years. There was no significant difference in those starting azathioprine between responders and non-responders (75\% [23/32] vs $87.5 \%[21 / 24] ; \mathrm{p}=0.20)$, or in rates of infliximab $(22 \%[7 / 32]$ vs $37.5 \%$ [9/24], $p=0.24)$ or surgery $(28 \%$ [9/32] vs $37.5 \%$ [9/24], $\mathrm{p}=0.57$ ).

Conclusion This is the first study proving that achievement of clinical remission with EEN predicts an improved outcome for paediatric patients with Crohn's disease over the next 5 years. It is possible that this is due to improved mucosal healing in children responding to EEN.

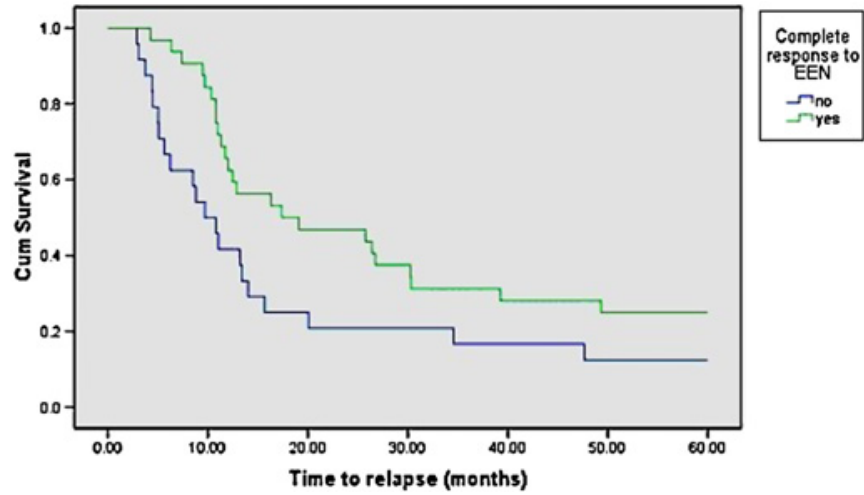

Abstract PTU-131 Figure 1 Time to relapse in responders and non-responders to EEN ( $p=0.041)$ [log ran test-Kaplan-Meier survival analysis].

A.Rao and N.Kamperidis contributed equally and should be considered as joint first authors.

Competing interests None declared.

\section{PTU-132 INCREASED DUODENAL INTRA EPITHELIAL LYMPHOCYTES (IELS) ARE ASSOCIATED WITH RECURRENT ABDOMINAL PAIN AND PARASITE INFECTION BUT NOT HELICOBACTER PYLORI IN A PAEDIATRIC CHILEAN COHORT}

doi:10.1136/gutjnl-2012-302514c.132

${ }^{1} \mathrm{M}$ M Walker, ${ }^{* 1} \mathrm{G}$ S Rakhra, ${ }^{2} \mathrm{~A}$ Villagran, ${ }^{2} \mathrm{P}$ R Harri, ${ }^{3} \mathrm{~J}$ E Crabtree. ${ }^{1}$ Histopathology, Imperial College, London, UK; ${ }^{2}$ Division of Pediatrics, School of Medicine Pontificia Universidad Catolica de Chile, Santiago, Chile, ${ }^{3}$ Leeds Institute Molecular Medicine, St. James's University Hospital, Leeds, UK

Introduction Functional Recurrent Abdominal Pain (RAP) is a paediatric functional gastrointestinal disorder with poorly investigated pathophysiology. Proposed aetiology varies and the diagnosis is characterised by Rome III criteria. Some studies consider Helicobacter pylori to be a cause of RAP, while others disagree. The aim of this study was to investigate upper gastrointestinal pathology in a cohort of 123 children in Chile with respect to RAP, $H$ pylori infection and other concurrent infection.

Methods This blinded retrospective and IRB-approved study analysed biopsies taken from the gastric antrum and body and the duodenum in 123 Chilean children referred to endoscopy (with informed parental consent). Histopathology was evaluated against a clinical database which included symptoms, symptom duration and endoscopy findings. Rome III criteria were used to assign RAP to the relevant cases. All patients had stool microbiology and parasitology. $H$ pylori infection was assessed by serology and histology. In the duodenum, routine histopathology and also eosinophil counts (in $5 \mathrm{HPF} \times 40$ magnification), were performed by microscopy. IELs/100 enterocytes were counted. Independently those patients with IELs $>25$ had serology performed for coeliac disease.

Results Overall 105 patients were diagnosed with RAP and 12 patients were able to act as controls, having no symptoms of RAP or concurrent infection. The Rome III diagnosis of RAP was significantly associated with higher IEL counts ( $>20$ in 74 patients) compared to controls $(p=0.04)$. Furthermore, a higher IEL count was also positively associated with parasitic infection (nine with parasites) $(p=0.02)$. Of 16 patients with lymphocytic duodenosis, (>25 IELs per 100 enterocytes) only three were infected by $H$ pylori. One had coeliac disease with positive serology. Antral nodularity was observed in association with lymphoid follicles $(p \leq 0.01)$ and H pylori infection $(p<0.01) .28 \%$ in this cohort were positive for $H$ pylori but infection was not 